\title{
Real Time Distance and Speed Evaluation Using Mobile Robot
}

${ }^{* 1}$ Rahul Gupta, ${ }^{2}$ Ramchand Hablani, ${ }^{3}$ Susmita Guin

${ }^{1,2,3}$ Shri Ramdeobaba College of Engineering and Management, Nagpur, Maharashtra, India

Email:*rahul37g@gmail.com, hablanir@ rknec.edu, guins@ rknec.edu

Received: 09 ${ }^{\text {th }}$ July 2018, Accepted: $14^{\text {th }}$ August 2018, Published: $31^{\text {st }}$ August 2018

\begin{abstract}
We all are aware that the future is of artificial intelligence and we want that machine should do smart work. Sometimes, due to hard work driver is not able to concentrate properly while driving the vehicle, also if driver is driving the vehicle continuously for a long time, he may suffer from fatigue in eyes and may not be able to focus on road properly, which may lead to accident if driver fails to detect the presence of obstacle. To overcome this problem, we need an automated vehicle, which can detect the obstacle and can calculate the distance along with the current relative speed of the vehicle and can perform the action accordingly.

In this paper, we introduce the Real Time Distance and Speed Evaluation (RTDSE) system using mobile robot which detects the presence of object before accident and calculate the distance between the robot and obstacle along with the current relative speed of the robot, which helps system to perform actions like reducing the speed of robot, overtaking the object or to apply emergency brakes.
\end{abstract}

Keywords: Obstacle Detection, Distance Measurement, Robot, Speed Measurement, Stereo Vision, ZED Camera.

\section{Introduction}

Due to increasing challenges in day to day life and to reach the destination on time, time plays an important factor. To save time, people drive vehicle fast on road and as a result, it leads to an accident. One of the main reasons is human error, which can be reduced but cannot be avoided completely. A human error like fatigue in eyes due to whole day hard work or by driving nonstop for a long time which makes driver exhaust and as a result, he fails to detect the obstacle or vehicle on the road which leads to an accident. If the driver is drunk, he may lose control over the vehicle and can cause damage to the vehicle or in the worst-case scenario may lead to a dangerous accident and as a result can cause his or others life at great risk.

Another reason is unexpected obstacles like an animal, pedestrian or another vehicle which suddenly appears in front of the vehicle and as a result driver gets dazed and fails to apply emergency brakes or lose control over vehicle due to the sudden appearance of obstacles.

To minimize this human error which is causing an accident, an automated system should be used which act as a driver assistance system for controlling the speed of the vehicle or to apply emergency brakes. To achieve the above-mentioned goals, we are proposing a Real Time Distance and Speed Evaluation (RTDSE) system. This system will detect obstacles in front of the robot and avoid it, with short response time. Our aim is to eliminate the cause of accident produced by human error by introducing RTDSE using the mobile robot. The system collects information from various types of sensors mounted on the robot and performs computation to detect if any obstacle is present. If the difference between the speeds of both robot and object is significant then the system will apply the brakes or bypass the obstacle.

The work presented here was mostly concentrated on the robot to perform as a smart robot on roads. We used Fire Bird VI Robot which has an inbuilt array of sensors (Fig. 1). The vehicle at the front side of the robot is named as a preceding vehicle (object). The Fire Bird VI Robot is equipped with actuating devices along with inbuilt sensors of firebird VI. Two IR range and ultrasonic sensors are fixed at the front side, two sensors on either side and two sensors on the back side to detect the presence of an object (for improving accuracy in case of foggy weather).

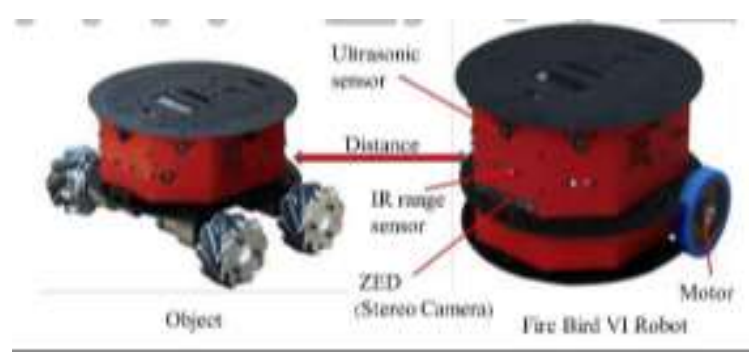

Figure 1. Schematic Showing General Representation of RTDSE

We are measuring distance and speed using stereo vision method. For stereo vision, ZED camera is being used, which is attached to the front side of the robot. There should always be enough distance for braking or controlling the speed required by the robot at current speed. The correction needed in the speed of the robot was computed by providing the current speed as a feedback signal to the control system.

\section{Related Work}

In this section, we put forth some of the related research that has been done in this field. Sheorey and Shrivas [1] proposed a method by fusing two low ranges of ultrasonic sensors and a proximity sensor for accumulating information about the speed and the position of the preceding vehicle from the host vehicle. Depending on the system hardware, the speed and the response time of the corresponding 
host vehicle, they guaranteed safety by performing safe evaluation, semi-critical and critical zones.

Marshall, Ian, and Viet [3] coupled two sonars and a camera. Color based segmentation was used in order to detect the obstacles and if segmentation algorithm fails, sonars are used to find the direction of the movement. However, fails to distinguish the obstacles and the ground surface if both are of the same color. Ohya, Yuta, and Soumare [4] made use of laser projector along with a stereo vision system in order to detect objects on the highway. The laser is used for calculation of the distance between the object and the robot. Light filters were employed on the cameras for capturing the reflected lasers. The above approaches were designed to work in an indoor environment and hence fails when exposed to the outdoor environment.

FranCois Marmoiton, et al. [5] have used camera sensor for providing information about the obstacles and driving lanes. The relative speed calculation was done with the help of monocular vision. However, they never got an accurate result in their test.

Avinash N., et al. [6] have used machine vision camera to calculate speed by dividing the distance covered by the time taken. On each scene in each frame they segmented the object and applied image metrology principle for depth calculation of segmented object.

\section{Proposed Method}

The proposed system will detect the presence of an obstacle in front of the robot, measures the distance between the robot and the obstacle along with the current speed of the robot and perform an action like braking or control speed. To increase the accuracy and robustness of the system along with ultrasonic sensor, we are also using the stereo camera for measuring the distance of objects ahead. RTDSE was formulated by considering the distance of the preceding vehicle and the current speed of the robot. Current speed signal was used as a feedback signal to minimize the error. Finally, an errorless output signal was generated, for which the feedback speed of the robot was compared with the input speed signal.

\section{A. Sensing and actuation}

The input system of RTDSE involves of a stereo camera, IR range, and ultrasonic sensors and rotary encoder. Fig. 2 depicts the input sensors and camera. All the input signals (I1, I2, I3, and I4) received by the input block were given to the electronic control unit. The stereo camera was fixed at the front center of the robot. It is used to feed the input to RTDSE as soon as it detects the presence of preceding object and measures the distance and relative speed between them. Two IR range and two ultrasonic sensors were mounted on the front side, two on the back side and two on both (left and right) sides. Its function is detecting obstacles, to improve the accuracy of detection and to avoid a collision from all sides of the robot.

Fig. 2 also shows the connection of control unit with the output devices. The output signals (O1 and O2) were received from the control unit after analyzing the input signal and performing an operation on it. The actuation system used was DC motor. It keeps running at a constant speed unless control action is initiated and as an action, the speed of robot was brought to zero or decreased depending upon the distance and relative speed of the robot.

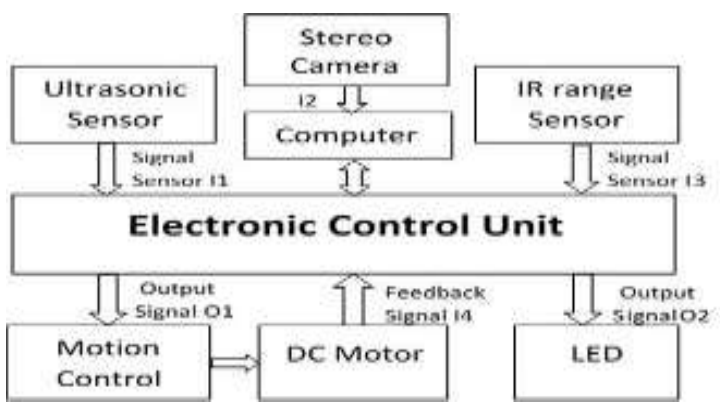

Figure 2. Schematic Showing Arrangement of Camera and Actuators

The robot has a dedicated motion control unit for controlling motors in open loop, closed loop velocity, and closed-loop position control mode. In case of braking, LED was used as a warning signal for braking to indicate the vehicle following the robot from the back side. The relative speed of the robot and the distance [2] lead to the output actuation in the form-controlled action.

\section{B. Fire bird VI Robot}

Firebird VI [7] is used for advanced research in mobile robotics, as it is a versatile, rough and reliable robot. It has a quadrature position encoder with highperformance gear motors. Speed and acceleration control with smart motion controller is used to drive the encoder. The robot can be operated inside or outside environment, just by selecting the right motion drive.

A variety of sensors, for example, eight ultrasonic range sensors with a range of 6 meters and 360degree range, eight IR distance range sensor which has a sensing range of 4 to $30 \mathrm{~cm}$. It is used to cover the blind spot of the ultrasonic range sensor and stereo camera. For communication Fire Bird VI supports two different modes, viz. $2.4 \mathrm{GHz}$ (wireless) module and USB (Serial).

\section{ZED stereo camera}

The ZED is a stereo camera [8], [10] that replicates the way human vision works. Making use of its two "eyes" and through triangulation, the ZED gives a 3D comprehension of the scene, it captures, allowing your application to be more aware of the surrounding space and motion.

Utilizing cutting-edge technology based on human stereo vision, the ZED camera includes 3D mapping, depth observation and positional tracking to any application. Since ZED visualizes the world in three dimensions, by making use of binocular vision and 
high-resolution sensors, it can detect the object from 0.5 to 20 meters.

\section{Depth from Stereo:}

The working of ZED camera is similar as the binocular vision. On an average of $65 \mathrm{~mm}$, human eyes are horizontally separated. In this manner, each eye has a somewhat unique perspective of the world around. By looking at these two prospects, human brain is able to estimate depth as well as 3D movement in space. Similarly, ZED camera consists of two eyes which are 12 $\mathrm{cm}$ separated from each other. It is able to capture highresolution three-dimensional videos of the given section or clip. It also estimates the motion and depth by relating the displaced pixels between the right and left images.

\section{Depth map}

The ZED stores a value of distance $(Z)$ for every pixel (X, $\mathrm{Y})$ in the image. The distance is generally calculated from the back of the left eye of the camera to the scene object. Depth maps calculated by the ZED are encoded on 32 bits so they cannot be shown directly. Depth map can be represented as monochrome (grayscale) 8-bit representation which has values between $[0,255]$, where 255 denotes the nearest conceivable depth value and 0 denotes the most distant possible depth value.

\section{3-D Point Cloud}

Depth information is also represented by a 3-D point cloud. A point cloud can be viewed as a depth map in three dimensions. While a depth map contains the distance or $\mathrm{Z}$ data for every pixel, a point cloud is a collection of $3 \mathrm{D}$ points $(\mathrm{X}, \mathrm{Y}$, and $\mathrm{Z})$ that denotes the outer surface of the scene and can contain information of color.

\section{Distance calculation}

\section{1) Measuring distance in the point cloud}

When estimating distance, 3D point cloud [8] is used rather than the depth map. The distance of the object with respect to the left eye of the camera is computed by the following Euclidean distance formula:

$D=\sqrt{x^{2}+y^{2}+z^{2}}$

The distance is measured in millimeters $(\mathrm{mm})$ and $1080 \mathrm{p}$ video mode along with 30 frames per second (fps) and the $3840 \times 1080$ resolution were used for calculations.

\section{2) Ultrasonic Sensor}

To calculate the distance from the ultrasonic sensor, short burst is transmitted from the transmitter which gets reflected back by the target and is received by the receiver. The time required for transmission and reception is calculated by the speed of sound and Speed equal to distance divided by time equation.

\section{E. Speed calculation}

Speed is the ratio of distance divided by time. Speed is directly proportional to distance while inversely proportional to time. We can also define speed as a rate of movement. Speed is a scalar quantity and velocity is the proportional vector amount of speed, but it does not consist in the element of direction that velocity consists.

The value of speed is calculated by dividing the distance by the time. To calculate the speed of movable object, one should know the distance carried and the time taken to cover the distance.

Progression of steady picture or images is called a video sequence which is produced at distinct time occasions. This image is considered as sequence of frames. To get the knowledge about how much the farther object has shifted in progressive video frames, it is required to segment the object in each picture in each frame. Then calculate the depth of the particular segmented object in the picture, once the metrology rule is utilized. Depth estimation is applied on every frame. Measurements of distance for the detected object from a frame to another is computed by performing subtraction of the estimated depth from given frames.

\section{Relative Speed}

The speed of one moving object (object A) with respect to another moving object (object B) is called relative speed. Relative speed will [6] be additive if object $\mathrm{A}$ and $\mathrm{B}$ are moving in the same direction and subtractive if object A and B are moving opposite to each other. Since here the frame rate is proportional to time (30 fps is frame rate having an interval between frames 1/30 seconds). Also, speed equation can be changed to use the frame rate directly. Hence, the object can be seen in successive frames.

Speed $=($ Travelled Distance in one Frame $X$ Frame Rate)

Here speed is evaluated in millimeters per seconds $(\mathrm{mm} / \mathrm{s})$, a distance in one frame is in millimeter while frame rate is measured in frames per second (fps) [6], [9]. From camera position and by using the distance calculation formula, we can calculate the distance traveled in each frame. Therefore, there is a need to calculate the difference in depth over the successive frames to measure the distance which is covered by moving object in the video.

We have seen in kinematics, the difference in a vector of velocities of two objects is known as relative velocity, which has been evaluated in terms of a single coordinate system, usually an initial frame of reference until specifically stated.

For example, let's assume a velocity of particle A is VA and particle B is VB in terms of a specified initial coordinate system. Then, the relative velocity of $\mathrm{A}$ with respect to B or $V_{A \text { rel } B}$ is given as

$V_{\text {Arel } B}=V_{A}-V_{B}$

Conversely, the velocity of $\mathrm{B}$ relative to $\mathrm{A}$ is

$V_{B \text { rel } A}=V_{B}-V_{A}$ 


\section{Position Calculation}

Calculation of position encoder resolution:

Robot [7] is moving forward or backward (encoder resolution is in $\mathrm{mm})$.

Wheel diameter: $\mathrm{d}(\mathrm{mm})$

\section{Wheel circumference (C): $d * \pi$}

No. of counts in 1 rotation of output shaft: $\mathrm{N}=3840$

No. of counts per $1 \mathrm{~mm}$ distance traveled $(\mathrm{Y})=N / C(6)$

Distance traveled per count $=C / N(\mathrm{~mm})$

\section{Experiment and Result}

The robot used in RTDSE was driven by DC motor, wheels $100 \mathrm{~mm}$ diameter and $20 \mathrm{~mm}$ wide. For the realtime testing purpose, the input speed was limited to a safe value. The safe velocity of the robot is $82 \mathrm{~mm} / \mathrm{s}$. $12 \mathrm{~V} \mathrm{DC}$ motor was used, which gives 100 RPM for driving the robot. The robot can run on the different speed required for testing. As per requirement for real-time testing, eight ultrasonic sensors, eight IR distance range sensor and stereo lab's ZED camera are used in the proposed system. The resolution of the camera used is $3840 \times 1080$ with 30 frames per second (fps). USB 3.0 is used for fast data transfer from a stereo camera to the computer.

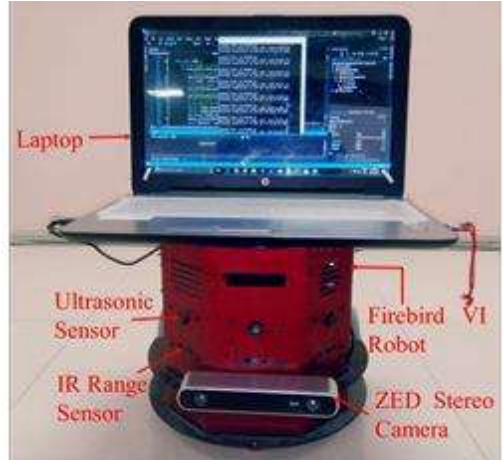

Figure 3. Mobile Robot and Hardware Setup

Fig. 3 show Fire Bird VI robot with sensors along with ZED stereo camera attached to it. A laptop was placed on the robot in order to process the images captured by the ZED camera. For object detection, distance and speed estimation, a combination of ZED SDK, CUDA, and OpenCV in Visual Studio are used. For building libraries and linkers, CMake is utilized for above software not only in release mode but also in the debug mode. Real time testing has been achieved by running a remote car in front of the robot and for communication, USB serial port was used for communication between the robot and the laptop. For calculation, we selected three different objects of different size and shape. For measuring distance, we placed the object at a certain distance which was measured by a ruler in millimeter and then we calculated the distance between objects which was stationary by the stereo camera and ultrasonic sensor. We got a slight difference reading from the sensor and ZED camera. Reason for variation in distance measured by ZED is due to different reflective index. The results obtained from the sensor and the camera along with error is shown in Table I.

After that, we run the robot at a different speed with various test and measured the distance between the object from stereo camera and ultrasonic sensors.

We have total three methods for measuring the speed of the robot.

1. Manual speed measurement.

2. Speed measurements using wheel encoder.

3. Speed measurement using the stereo camera.

To measure the speed manually, we have measured distance with ruler and time with a stopwatch and then to calculate speed, we used formula

Speed $=\frac{\text { Distance }}{\text { Time }}$

For measuring speed using speed encoder, we have programmed a robot to move at the speed of 82 $\mathrm{mm} / \mathrm{s}$. The robot was moved by $1800 \mathrm{~mm}$ and the time required to cover this distance was 21.95 seconds. So, this gives us the speed of $82 \mathrm{~mm} / \mathrm{s}$.

Now lastly to measure speed via ZED, we placed the camera stationary and run the robot at $82 \mathrm{~mm} / \mathrm{s}$ towards the camera. We set the camera resolution mode at 1080 with $30 \mathrm{fps}$ but during test we observed that it is only working at 15 fps because of large computation work. We programmed ZED to capture 100 images and it took 6.852 seconds for this task. It took 100 readings of distance. The average difference of distance between two frames is 5.61 $\mathrm{mm}$ denoted as $\Delta x$ and time interval between two frames is 0.06852 seconds denoted as $\Delta t$.

Velocity $=\frac{\Delta x}{\Delta t}$

The speed evaluated is denoted as millimeter per second $(\mathrm{mm} / \mathrm{s})$.

\begin{tabular}{|c|c|c|c|c|c|}
\hline Sr. No. & $\begin{array}{c}\text { Real } \\
\text { distance } \\
(\mathrm{MM})\end{array}$ & $\begin{array}{c}\text { ZED } \\
(\mathrm{mm})\end{array}$ & $\begin{array}{l}\text { Error } \\
\%\end{array}$ & $\begin{array}{c}\text { Ultrasonic } \\
\text { Sensor } \\
(\mathrm{mm})\end{array}$ & $\begin{array}{l}\text { Error } \\
\%\end{array}$ \\
\cline { 2 - 6 } & 1050 & 990 & 5.7 & 1040 & 0.9 \\
\hline 2 & 1250 & 1180 & 5.6 & 1239 & 0.8 \\
\hline 3 & 3000 & 2900 & 3.3 & 2991 & 0.3 \\
\hline \hline
\end{tabular}

Table 1: Distance Estimation at Different

Position

\begin{tabular}{|c|c|c|c|c|}
\hline \multirow{2}{*}{ Sr. No. } & $\begin{array}{c}\text { Manual } \\
\text { Estimation } \\
(\mathrm{mm} / \mathrm{s})\end{array}$ & $\begin{array}{c}\text { Encoder } \\
\text { Estimation } \\
(\mathrm{mm} / \mathrm{s})\end{array}$ & $\begin{array}{c}\text { ZED } \\
\text { Estimation } \\
(\mathrm{mm} / \mathrm{s})\end{array}$ & $\begin{array}{c}\text { Relative } \\
\text { Speed } \\
(\mathrm{mm} / \mathrm{s})\end{array}$ \\
\hline 1 & 82 & 82 & 81.8 & 18 \\
\hline 2 & 250 & 250 & 248.1 & 150 \\
\hline \hline
\end{tabular}

Table 2: Speed Estimation at a Different Position 
We got variation in speed measured by ZED. Reason for variation in speed may be due to missing of frames while capturing. The result obtained from manual, wheel encoder and stereo camera testing is shown in Table II.

To measure the relative speed, the robot was operated at a safe velocity of $82 \mathrm{~mm} / \mathrm{s}$ and speed of another moving object was $100 \mathrm{~mm} / \mathrm{s}$. Then the relative speed calculation was done as described in the relative speed section. The results obtained in the test are shown in Table II.

\section{Conclusion and Future Work}

In this paper, we presented a real-time, distance and speed evaluation using the mobile robot system, to detect the object by using stereo vision method with the help of ZED camera mounted on the robot. Further, we have also calculated the distance of the moving object along with the relative speed.

A new framework was developed for the RTDSE which makes the use of stereo camera, ultrasonic and IR range sensors to get the information about the position of objects and relative speed of the vehicle. Testing of the proposed algorithm on higher vehicle speed by using the stereo camera or by employing longrange sensors and hardware components having higher computing speed and faster response time has also been done. The proposed method is able to take appropriate control action if it finds any static or moving object. RTDSE was able to avoid a collision in all cases tested in the real environment. However, there is still a room for improvement. Our future work includes improvement of the system performance by applying Machine learning methods such as supervised learning algorithms. It will not only improve the efficiency of the system but also simplify the work by detecting the given object accurately. We will try to develop a classifier which will take useful features to detect an object from its background.

\section{References}

[1] Tanuja Sheorey, Nikhil Vivek Shrivas, "Development of Sensor Based Front End Collision Avoidance System for Highways", International Conference on Information and Automation Lijiang, China, August 2015.

[2] Jiangfeng Wang; Feng Gao; Shaoyou Shi; Jian Wang, "A New Method for Distance and Relative Velocity Measurement in Vehicle Collision Warning System", Intelligent Systems Design and Applications, 2006. ISDA'06. Sixth International Conference, vol.1, pp.1142, 1147, 16-18 October 2006.

[3] Viet, C. N., Marshall, Ian., "Vision-Based Obstacle Avoidance for a Small, Low-Cost Robot" International Conference on Advanced Robotics, 2007, pp 275-279

[4] Soumare, S., Ohya, A. and Yuta S., "Real-Time Obstacle Avoidance by an Autonomous Mobile
Robot using an Active Vision Sensor and a Vertically Emitted Laser Slit" Intelligent Autonomous Systems 7, 2002, pp 301-308

[5] FranCois Marmoiton, FranCois Collange and Jean Pierre DCrutin, "Location and relative speed estimation of vehicles by a monocular vision", Intelligent Vehicles Symposium (IV), Dearborn, MI, USA, 5-5 Oct. 2000

[6] Avinash N., Dev Dutt Yadav and Murali S., "Vision Based Relative Speed Detection of Moving Objects in a Traffic Environment", Information and Multimedia Technology, 2009.

[7] "Fire Bird VI NEX Robotics," [Online] Available: http://www.nexrobotics.com/products/fire-bird-vi-robot/firebird-vi-robotic-research-platform.html, April 2018.

[8] "Stereo Lab's ZEDCamera,"[Online]. Available: https://www.stereolabs.com/ zed/specs/, April 2018.

[9] Ishan kumar Shahu and Ramchand Hablani "An Object Detection, and Technique for Velocity Estimation along with Classification on the Basis of Speed", Int. Journal of Advanced Research in Computer Science and Software Engineering(IJARCSSE), vol.6Issue 5, May 2016.

[10] Ishan kumar Shahu and Ramchand Hablani "An Object Detection, and Parametric Classification- A Review", Int. Research Journal of Engineering and Technology (IRJET), vol.3-Issue 2, February 2016. (eISSN:2395-0056 p-ISSN:2395-0072) 